\title{
Fast Scanning Electron Diffraction and Electron Holography as Methods to Acquire Structural Information on $\mathrm{Au}_{102}(\mathrm{p}-\mathrm{MBA})_{44}$ Nanoclusters
}

\author{
J. Eduardo Ortega, Ulises Santiago, Alina Bruna, Diego Alducin, German Plascencia Villa, Robert L. \\ Whetten, Arturo Ponce and Miguel José-Yacamán
}

Department of Physics and Astronomy, University of Texas at San Antonio, San Antonio, Texas 78249, United States.

Electron diffraction analysis in Transmission Electron Microscopy (TEM) is one resourceful but problematic technique for the characterization of sensitive materials due the high intensity on the convergent beam. In the case of ligand-protected clusters there are two key damage mechanisms that can appear during the data acquisition of nanobeam diffraction patterns (NBD) in the TEM: (i) the knock-on effect, arising from the momentum transfer between the electron beam and the nanoclusters and (ii) the ionization damage (radiolysis).

Low voltage $(80 \mathrm{kV})$, cryo-TEM, rapid acquisition in aberration-corrected high angle annular dark field (HAADF) STEM imaging and low dose TEM are some of the approaches that can be implemented in order to reduce radiation damage. The effectiveness of these methods are based on reducing the dose or reducing the exposition time. As the later directly affects the quality of imaging techniques is more suitable for interferometric methods. Herein we present low dose rapid nanobeam electron diffraction and electron holography analysis to characterize the $\mathrm{Au}_{102}(\mathrm{p}-\mathrm{MBA})_{44}$ nanocluster as both can reduce the electron dose exerted on the protected clusters.

The samples were synthesized by the two-phase transfer method and evaluated by non-denaturing polyacrylamide gel electrophoresis (PAGE). The acquisition of ultra-low dose diffraction patterns was made using a JEOL $2010 \mathrm{~F}$ operating at $200 \mathrm{kV}$, and a probe size of $3 \mathrm{~nm}$, to which a NAMOMEGAS ASTAR system has been coupled, allowing the fast scan of the electron beam on a pre-established area, followed by the collection of the NBD patterns. The system is equipped with an ultrafast TVIPS 16mega pixel F416 CMOS camera with a dynamic range of 10000:1. The advantage of a CMOS camera sensor over a CCD relies not only in its 16 bit resolution but also on the streaking effect suffered by the CCD sensors. Electron holography was carried out in a JEOL ARM 200F operated at $200 \mathrm{kV}$. Off-axis EH was performed under Lorentz conditions, where the imaging is achieved using the objective minilens.

For the digital examination of the patterns, we have implemented an orthogonal supercell size, whose dimensions of $36 \times 36 \times 18 \AA$, in order to evade periodicity effects. To match the expected diffraction data with the experimental ones is important to consider that the particle can rotate over its own axis and produce a different projection. Taking the five-fold symmetry structure as the origin of the $\mathrm{z}$ orientation in the coordinate system the nanoparticle is rotated on one degree steps in five different directions: $x, y$, $x y, 1 / 2 x y, x 1 / 2 y$, in order to obtain a data set of 900 simulated diffraction patterns where the main fitting parameter is the relationship between spot positions and angles [1]. The experimental and simulated patterns were deconvoluted using an iterative Richardson - Lucy algorithm [2, 3] implemented in the G'MIC program [4]. As can be observed on Figure 1 from the raw diffraction pattern obtained with our setup is possible to extract and enhance the diffraction pattern that serves to extract the structural data from the nanoparticles core. 
For the experimental holograms, our analysis is based on the sensitivity of EH to the mean inner potential, (i.e. volume-averaged electrostatic contribution of the crystal potential). We used EH to reconstruct the phase shift induced by our metallic clusters. Retrieving the phase information form the holograms, the mean inner potential can be derived for each particle and later on arranged as a function of the core size. According to previous work of Popescu et al. [5] this value exhibits a large increase for sizes below $2 \mathrm{~nm}$ which increases the sensitivity of our analysis range, making possible to validate the particles that match the threshold value for gold cores of this size [6].

\section{References:}

[1] Alina Bruna et al. J. Phys. Chem. C 120, (2016), p. 1902-1908

[2] Richardson WH. JOSA 62, (1972), p. 55-59.

[3] Lucy LB. Astron. J. 79, (1974), p. 745-754.

[4] GREYC's Magic Image Converter home page. http://gmic.eu (accessed: 9, 2015).

[5] Radian Popescu et al, Physical Review B 85, (2012), p. 045443.

[6] This project was supported by grants from the National Center for Research Resources (5 G12RR013646-12), the National Institute on Minority Health and Health Disparities (G12MD007591) from the National Institutes of Health and the Welch Foundation (grant No. AX-1615 and AZ-1857). The authors acknowledge: Conacyt \# 250836, Dr. D. Black for performing the ESI-MS data.

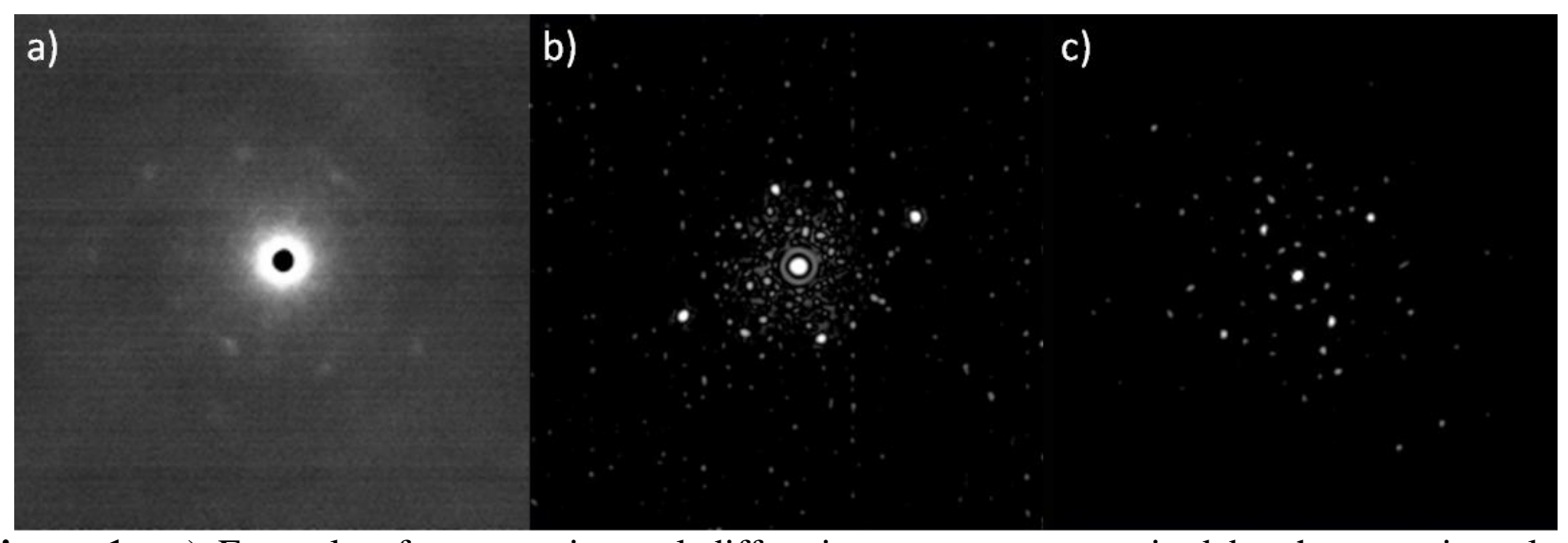

Figure 1. a) Example of an experimental diffraction pattern, as acquired by the scanning electron diffraction setup; b) A filtered diffraction pattern, for which a Richardson-Lucy algorithm has been applied, with further filtering based on Gaussian differences to enhance contrast; c) The simulated pattern from the metallic gold nanoclusters. 